\title{
Fenretinide (4-HPR): A Preventive Chance for Women at Genetic and Familial Risk?
}

\author{
Massimiliano Cazzaniga, Clara Varricchio, Chiara Montefrancesco, \\ Irene Feroce, and Aliana Guerrieri-Gonzaga \\ Division of Cancer Prevention and Genetics, European Institute of Oncology, 20141 Milan, Italy \\ Correspondence should be addressed to Massimiliano Cazzaniga, massimiliano.cazzaniga@ieo.it
}

Received 5 October 2011; Revised 12 December 2011; Accepted 15 December 2011

Academic Editor: Dianne R. Soprano

Copyright ( 92012 Massimiliano Cazzaniga et al. This is an open access article distributed under the Creative Commons Attribution License, which permits unrestricted use, distribution, and reproduction in any medium, provided the original work is properly cited.

The incidence and mortality of breast cancer have been recently influenced by several new therapeutic strategies. In particular our knowledge on cancer precursors, risk biomarkers, and genetics has considerably increased, and prevention strategies are being successfully explored. Since their discovery, retinoids, the natural and synthetic derivatives of vitamin A, have been known to play a crucial role in cell and tissue differentiation and their ability to inhibit carcinogenesis has made them the ideal chemopreventive agents studied in several preclinical and clinical trials. Fenretinide (4-HPR) is the most studied retinoid in breast cancer chemoprevention clinical trials due to its selective accumulation in breast tissue and its favorable toxicological profile. This agent showed a significative reduction of the incidence of second breast tumors in premenopausal women confirmed after 15year followups. Considering Fenretinide protective action, a similar trend on ovarian cancer, this drug warrants reevaluations as a preventive agent for high-risk young women, such as BRCA-1 and 2 mutation carriers or with a high familial risk. This favorable effect therefore provides a strong rationale for a primary prevention trial in these unaffected cohort of women.

\section{Introduction}

In Western countries, breast cancer is a major concern and its incidence and mortality rates have been recently influenced by new therapeutic strategies. Our knowledge on cancer precursors, risk biomarkers, and cancer genetics has considerably increased, and prevention strategies are being successfully explored. Unfortunately, over the last decade, breast cancer prevention has been mainly focused on endocrine therapies using selective estrogen receptors modulators (SERMs) and aromatase inhibitors (AIs). Available preventive strategies for nonhormonal breast malignancies, more frequently expressed in BRCA mutation carriers and, in general, in high-risk population, are needed. For these reasons, a great number of novel chemopreventative agents are currently under investigation in order to evaluate their efficacy in this particular cohort of patients.

\section{Retinoids}

In accordance with their recognized role in the regulation of cell growth, differentiation, apoptosis, and their recognized inhibitory effect on cell growth in ER positive and negative breast cancer cells, retinoids (either natural or synthetic compounds structurally related to vitamin A) have long been studied for their chemotherapeutic effect and for their chemopreventive potential in breast cancer setting. Only recently, retinoids have also been applied in this unaffected high-risk population and they have demonstrated to be able to suppress tumor promotion and modify some properties of fully transformed malignant cells by activating and/or repressing specific genes [1]. Retinoids initiate ligandinduced dimerization of retinoid acid receptors $(\operatorname{RAR} \alpha, \beta$, and $\gamma$ ) and retinoid $\mathrm{X}$ receptors $(\operatorname{RXR} \alpha, \beta$, and $\gamma)$. Subsequently, receptors bind to retinoid response elements on 
DNA, and they initiate transactivation of retinoids response target genes [2]. Retinoid receptors are expressed in both normal and malignant breast epithelial cells and are critical for normal development. The mechanism by which retinoids inhibit breast cell growth has not been completely elucidated yet. Given the role played by RAR- $\beta$ in the carcinogenesis of different tumors, its regulation by retinoids has also been advocated as a putative mechanism of action of these agents [3]. However, they have been shown to affect multiple signal transduction pathways, including IGF, TGF $\beta$, and AP-1dependent pathways [4-8], as showed in Figure 1.

Several preclinical models suggest that retinoids inhibit mammary carcinogenesis in carcinogen-treated rats and in transgenic mice [9-11]. Recently, in order to reduce retinoids' side effects, RXR-selective retinoids, commonly known as rexinoids, have been studied as cancer preventive agents. In particular, preclinical studies have demonstrated that these compounds maintain retinoids' chemopreventive effect, but have greatly reduced toxicity [12]. 9cRA, a retinoid binding both RAR and RXR, has significantly delayed the ERnegative tumor development in SV40 tag mice and MNUtreated rats [13], although it induced significant cutaneous toxicity. In contrast, a RXR-selective retinoid, LGD1069, or bexarotene (Targretin), has suppressed both ER-positive and ER-negative tumor development with minimal toxicity $[14,15]$.

\section{Fenretinide (4-HPR)}

One of the most promising retinoids to be used in chemoprevention trials is the synthetic amide of retinoic acid fenretinide, $N$-4-hydroxyphenyl retinamide (4-HPR) (Figure 2).

Fenretinide was first synthesized by Gander in the late 1960s in the United States. Its biologic activity was assayed by Sporn et al., who also showed the preferential accumulation of this drug in the breast, instead of in the liver [30]. The inhibition of chemically induced mammary carcinoma in rats by fenretinide was first described in 1979 [31]. Since then, promising in vitro data and a favorable toxicity profile compared with that of other retinoids have led to the extensive study of fenretinide in chemoprevention trials targeting different organs [32].

Fenretinide has been found to have significant chemopreventive action in a large variety of in vitro and in vivo systems. Both fenretinide and its major metabolite, 4-metoxyphenyl retinamide (MPR), selectively accumulate in the human breast [33]. It should be noticed that some fenretinide-based toxicities could be due also to its hydrolysis so that it returns to retinoic acid in vivo [34]. Nevertheless, it remains a fascinating candidate for breast cancer chemoprevention.

\section{Mechanism of Action}

High-dose fenretinide is cytotoxic for a variety of different tumor cells in preclinical studies [35-37], although its accurate mechanism of action is not yet completely understood. However, it has been proposed that it might exert its inhibitory effects by means of both receptor-dependent and -independent mechanism (Table 1, [16-18]).

Although RAR influence on fenretinide action is highly debated, recent evidence would support the hypothesis according to which a mechanism does not require such relationship. In particular, we would stress the importance of the studies carried out by Giandomenico et al. [19], explaining the role of the stable expression of the dominant negative RAR $\alpha$; by Anding et al. [20], showing that the use of a RAR panantagonist influences an unhydrolyzable analogue of 4-HPR by inducing apoptosis with an independent RAR signaling pathway; by $\mathrm{D}^{\prime}$ Elia et al. [21], assuming resistance to differential responsiveness is present in different cell lines thus indicating that Fenretinide may act through different receptor types. All of these findings seem to diminish the importance of RAR role.

Fenretinide characteristic feature is the ability to inhibit cell growth through the induction of apoptosis rather than differentiation, an effect that is strikingly different from that of the all-trans retinoic acid [22]. Moreover, 4-HPRmediated apoptosis seems to be tissue-specific, so that multiple mechanisms might operate within specific tissues [17]. For example, in ovarian carcinoma cell lines, retinoids may induce apoptosis through the depolarization of the mitochondrial membrane and activation of caspase pathway $[23,24]$, while in the breast and in others cell lines apoptosis seems to be related with a direct molecular interaction with tubulin [25]. Moreover, reactive oxygen species (ROS), such as hydrogen peroxide and superoxide, seem to be critical in mediating apoptosis in different cancer cell types [2628]. The ability to increase ROS levels, in particular nitric oxide (NO) by NO synthases (NOS) over the elevation of sphingolipid ceramide levels [29], has been suggested as an explanation of the apoptotic effect of fenretinide. Recently, fenretinide has been shown to be able to induce NO-mediated apoptosis in breast cancer (BRCA-1)-mutated breast cancer cells [42].

Additional mechanisms are under investigations, such as the ability to inhibit cell growth by reducing the expression of growth-stimulating factors or by inducing the expression of growth-inhibitory factors.

A recent proposed surrogate biomarker of fenretinide efficacy is circulating insulin-like growth factor 1 (IGF1). The IGF system plays a pivotal role in cell proliferation of both epithelial and mesenchymal tissues by stimulating mitosis, protecting cells from apoptosis, and maintaining transformed phenotype [43]. Large prospective studies have shown that high circulating levels of IGF1 and lower levels of its major binding protein (IGFBP-3) are associated with a higher risk of developing subsequent premenopausal breast cancer and prostate, lung, and colorectal cancer [44-47]. This indicates that circulating IGF1 is a key regulator of cell and tumor proliferation for the vast majority of human epithelial cancer. Fenretinide has been shown to inhibit IGF1-stimulated growth of breast cancer cell lines (BCCLs) and to downregulate the IGF system in both ER-positive and ER-negative BCCL [48]. In addition, fenretinide reduces plasma IGF1 in early breast cancer [49]. The expression of HER2 has been recently observed to reduce fenretinide 


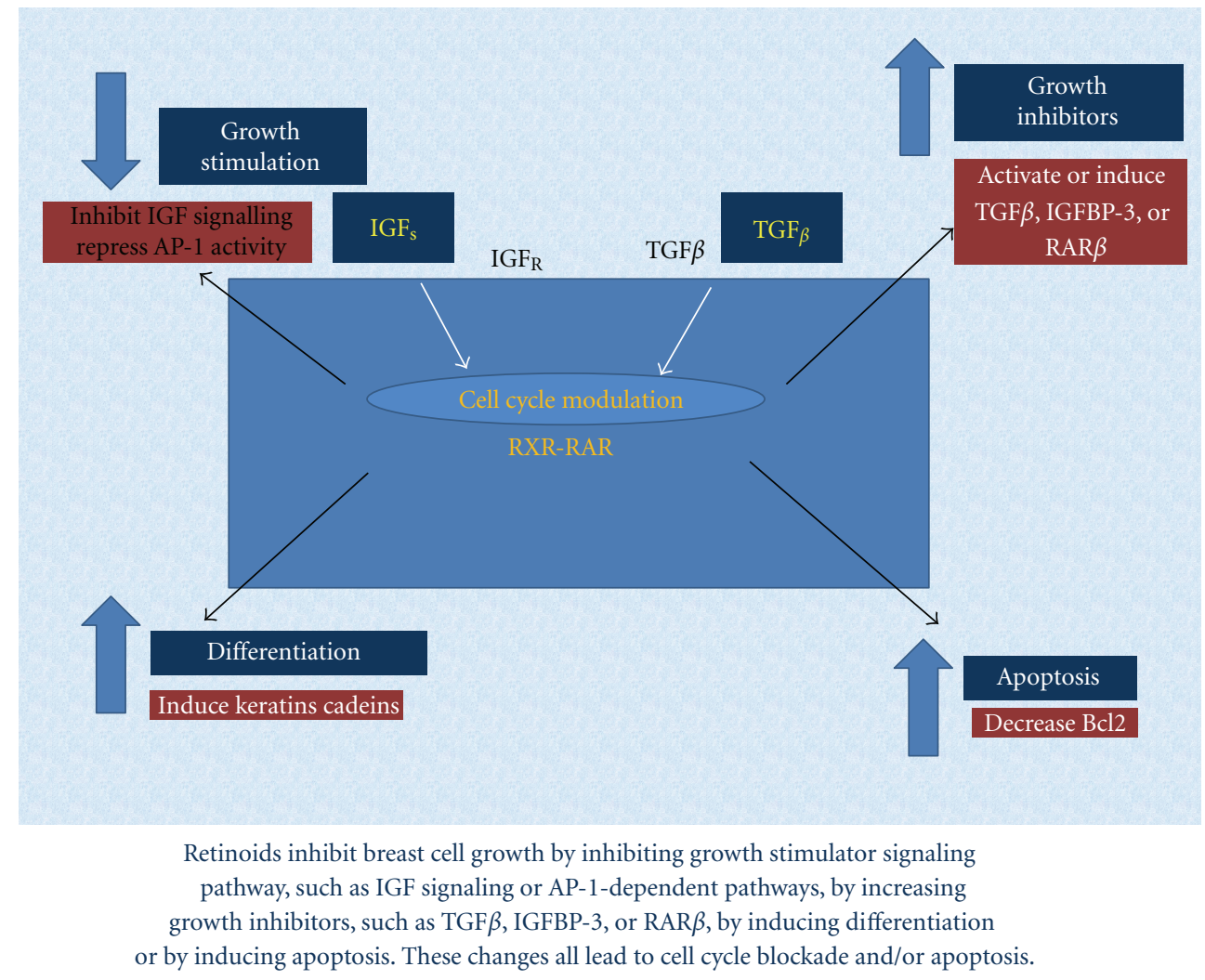

FIGURE 1: Retinoids' mechanism of action.<smiles>CC1=C(/C=C/C(C)=C/C=C/C(C)=C/C(=O)Nc2ccc(O)cc2)C(C)(C)CCC1</smiles>

Figure 2: Synthetic retinoid fenretinide.

ability to induce apoptosis in breast cancer cells. Moreover, researchers found that HER2 uses active human protein kinase (Akt) to induce cyclooxygenase (COX-2) expression and that inhibition of Akt or COX-2 increased 4-HPR induces apoptosis mediated by NO production [50]. Thus, a combination of 4-HPR with COX-2 inhibitors might be a new strategy to further investigate breast cancer chemoprevention.

\section{Pharmacology, Safety, and Tolerability}

Fenretinide pharmacokinetics and its possible side effects have been tested in several studies. The 5-year administration of the Milan study (see below) provided a vast corpus of information on the long-term safety and tolerability of this retinoid. As a major side effect, it induced a dose-related linear decrease of plasma retinol, associated with diminished retinal adaptation to darkness. In order to minimize this side effect, a 3-day treatment interruption at the end of each month was introduced to increase plasma retinol concentrations, allowing the partial recovery of retinal storage.

However, an accurate and complete evaluation of toxicity was hampered by the lack of a placebo control group. Dermatological, gastrointestinal, visual, and ophthalmologic events were relatively frequent, but were mostly mild. In a recent analysis of the phase III trial [51], the most common adverse events were diminished dark adaptation (cumulative incidence, 19\%) and dermatologic disorders $(18.6 \%)$, such as skin and mucosal dryness, pruritus, and urticaria. Less common events were gastrointestinal symptoms $(13 \%)$ and alterations of the ocular surface $(10.9 \%)$. Women in the control group complained of diminished dark adaptation, dermatologic disorders, gastrointestinal symptoms, and alterations of the ocular surface in $2.9 \%$, 
TABLE 1: Mechanism of action of Fenretinide (4-HPR). Corresponding references: [16-29].

\begin{tabular}{lc}
\hline Mechanism of action & Effect \\
\hline Receptor dependent & Regulation of growth, differentiation, and apoptosis \\
RAR- $\alpha, \beta, \gamma$ and RXR- $\alpha, \beta, \gamma$ (ligand-activated transcription factors) & \\
\hline Receptor independent & Apoptosis \\
Generation of ROS: hydrogen peroxide and superoxide & Cell growth inhibition \\
Induction of COX-2 expression by HER2/neu through activation of Akt & \\
Production of nitric oxide by NOS in BRCA 1 mutated breast cancer cells & \\
C-erbB-2 protein and mRNA downregulation & \\
Sphingolipid ceramide elevation and membrane synthesis alterations & Breast cancer development and progression \\
Mitochondrial damage with cytochrome-C release, disruption of & \\
mitochondrial transmembrane potential, and ROS generation & \\
Caspases activation & \\
\hline Induction of growth inhibition factors & \\
Inhibition of growth stimulating factors (IGF-1) & \\
Increased secretion of IGFBPs & \\
TGF- $\beta$ secretion & \\
\hline Decreased telomerase activity in MNU-induced mammary tumor and & \\
bronchial epithelium of cigarette smokers &
\end{tabular}

$2.9 \%, 5.4 \%$, and $3.2 \%$ of the cases, respectively. Interestingly, most side effects decreased with time and were significantly more frequent in postmenopausal women. Importantly, in contrast to other retinoids, prolonged administration of fenretinide is not associated with significant alterations of bone mineral density of the forearm [52]. However, a trend towards an increase in bone resorption markers suggests the need for further assessment at different skeletal sites.

After the completion of a phase I dose-ranging study [38], the $200 \mathrm{mg}$ daily dose was chosen as the safest dose for prevention, as one case of a pathological electroretinogram after a 24-week administration was observed with the $300 \mathrm{mg} /$ day dose [39]. Higher doses, up to $400 \mathrm{mg}$, have been used in women with metastatic cancer in combination with tamoxifen, with no evident toxicity on liver and lipid profile, but with an increased incidence of nyctalopia $[53,54]$. Peak levels of 4-HPR occur at approximately $6 \mathrm{~h}$ in adults with terminal half-life of approx. $13 \mathrm{~h}[39,55-57]$.

\section{Clinical Trials}

Since both 4-HPR and 4-MPR are selectively accumulated in the breast, evaluation of fenretinide as a chemopreventive agent in breast cancer has been particularly attractive. The most important clinical trials with fenretinide are mentioned in Table 2. As in the therapeutic setting, where drugs combinations are superior to monochemotherapy, the concept of combining agents with different mechanisms of action in the attempt to increase efficacy while minimizing side effects is a rational approach in chemoprevention. In preclinical models, combined administration of fenretinide and tamoxifen has proven additive and synergistic in both growth inhibition of MCF-7 cells and prevention of MNU-induced mammary carcinomas [58]. Moreover, the activity of 9-cis-retinoic acid against MNU-induced mammary tumors in Sprague-Dawley rats is enhanced by the combination with tamoxifen or raloxifene [11]. The safety and the tolerability of the combination of fenretinide and tamoxifen have been investigated in clinical trials in metastatic breast cancer patients [53] and in women at increased risk [59]. The concept of combining agents with different mechanisms of action in the attempt to increase efficacy on complementary molecular targets, while minimizing side effects is increasingly being pursued in breast cancer chemoprevention. A clinical randomized, double-blind, placebo-controlled phase IIb trial with a $2 \times 2$ factorial design to test this interaction (fenretinide and lowdose tamoxifen) was conducted at the European Institute of Oncology [60]. In spite of the favorable effects on plasma IGF-I levels and mammographic density, this combination did not reduce breast neoplastic events compared to placebo, whereas both single agents, particularly fenretinide, showed numerical reduction in annual odds of breast neoplasms.

Fenretinide (in combination with HRT) was also studied by our group in 226 postmenopausal healthy women, randomized in a two-by-two factorial design to either oral CEE $0.625 \mathrm{mg} /$ day or transdermal E2, $50 \mathrm{microg} /$ day and to fenretinide $100 \mathrm{mg} /$ twice a day or placebo for 12 months [61]. Oral CEE showed more favorable changes than transdermal E2 on circulating breast cancer risk biomarkers, while fenretinide exerted little modulation on most biomarkers.

The most important study where 4-HPR was administrated as a single agent is a multicentric phase III randomized trial, coordinated by the Istituto Nazionale dei Tumori in Milan, started in 1987. Stage I (T1-2 N0) breast cancer patients, aged 33-70 years, who had been operated on for breast cancer within the previous 10 years and had received no systemic adjuvant therapy were eligible [40]. Women were randomly assigned to receive either no treatment or fenretinide given orally at a dose of $200 \mathrm{mg}$ /day for 5 years. A placebo-control arm was not included in the study design because of the large capsule size and the objective nature of the main outcome measure. A 3-day drug stoppage at the end of each month was recommended in order to allow retinol recovery and to minimize dark adaptation impairment. The main outcome measure was the occurrence 
TABLE 2: Clinical trials with fenretinide.

\begin{tabular}{|c|c|c|c|c|c|}
\hline Study & Design & Treatment & End points & Outcomes & References \\
\hline Costa et al. (1989) & Phase I, R, PC (60) & $\begin{array}{l}\text { Orally: } 100,200 \text {, and } \\
300 \mathrm{mg} \times 6 \text { months } \\
\text { subsequently at } 200 \mathrm{mg} \\
\text { for another } 6 \text { months }\end{array}$ & Tolerability & $\begin{array}{l}\text { Recommended dose for } \\
\text { chemoprevention trials of HPR is } \\
200 \mathrm{mg} / \text { die. }\end{array}$ & {$[38]$} \\
\hline Formelli et al. (1989) & Phase II, R, PC (60) & $\begin{array}{l}\text { Orally: } 100,200 \text {, and } \\
300 \mathrm{mg} \times 6 \text { months } \\
\text { subsequently at } 200 \mathrm{mg} \\
\text { for another } 6 \text { months }\end{array}$ & Pharmacokinetic & $\begin{array}{l}\text { HPR treatment lowers retinol and } \\
\text { RPB plasma concentrations. This } \\
\text { effect is related to HPR levels and } \\
\text { is reversible on cessation of HPR } \\
\text { administration. }\end{array}$ & [39] \\
\hline Veronesi et al. (1999) & Phase III R (2867) & $\begin{array}{l}\text { Orally } 200 \mathrm{mg} \text { versus no } \\
\text { treatment } \times 5 \text { years }\end{array}$ & $\begin{array}{c}\text { Second breast } \\
\text { cancer prevention }\end{array}$ & $\begin{array}{l}\text { No statistically significant effect } \\
\text { but a possible benefit in } \\
\text { premenopausal women. }\end{array}$ & {$[40]$} \\
\hline Veronesi et al. (2006) & $\begin{array}{l}\text { Phase III, R, 15-year } \\
\text { followup (1879) }\end{array}$ & $\begin{array}{l}\text { Orally } 200 \text { mg versus no } \\
\text { treatment } \times 5 \text { years; } \\
15 \text {-year followup }\end{array}$ & $\begin{array}{c}\text { Second breast } \\
\text { cancer prevention }\end{array}$ & $\begin{array}{l}\text { 4-HPR induces a significant risk } \\
\text { reduction of second breast cancer } \\
\text { in premenopausal women, which } \\
\text { is remarkable at younger ages, and } \\
\text { persists several years after } \\
\text { treatment cessation. }\end{array}$ & {$[41]$} \\
\hline
\end{tabular}

HPR: Fenretinide; PC: placebo controlled; R: randomized; RBP: retinol-binding protein.

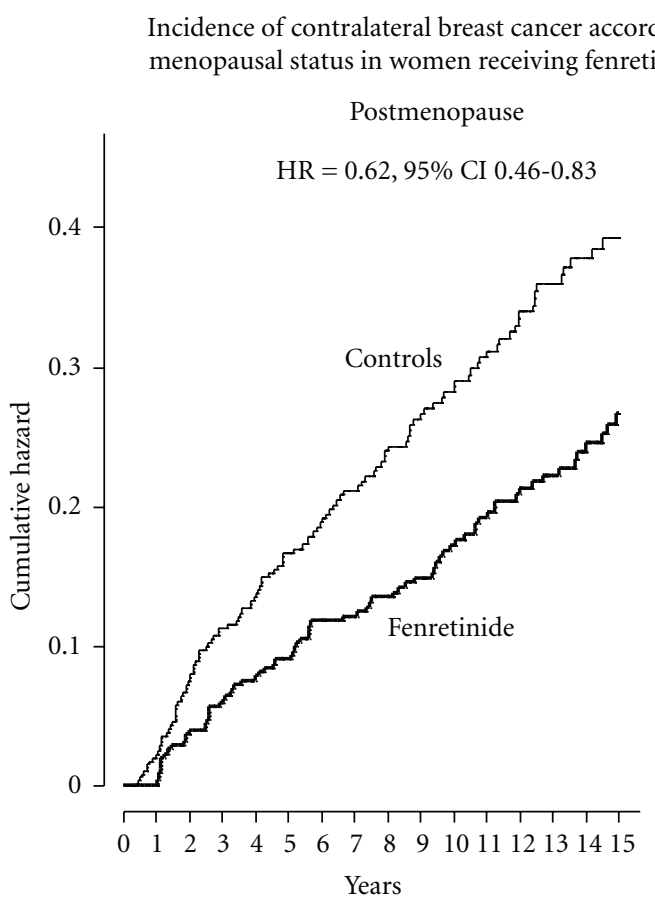

(a)
Incidence of contralateral breast cancer according to menopausal status in women receiving fenretinide

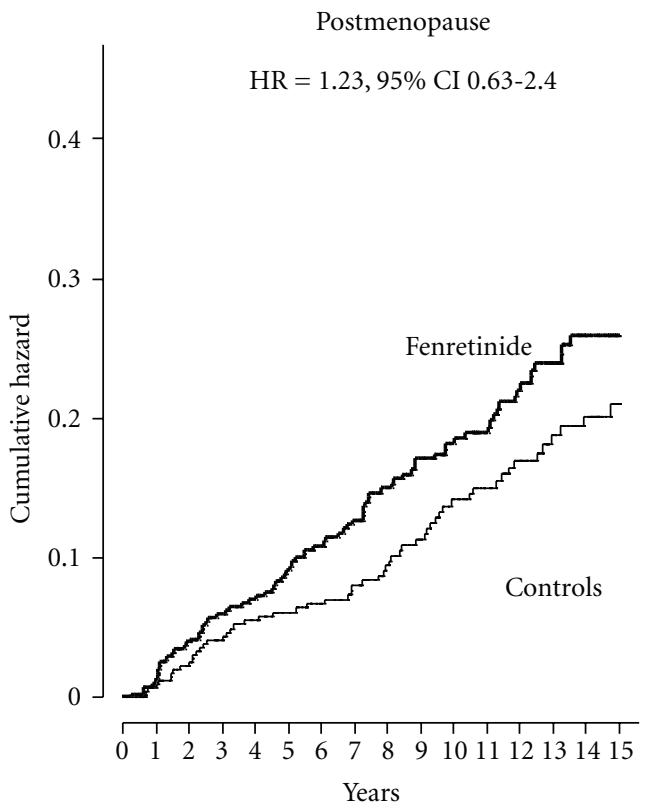

(b)

FIGURE 3: Opposite effect of fenretinide according to menopausal status. Cumulative hazard curves for all second breast cancers (contralateral and ipsilateral) by allocated arm, stratified by premenopausal women (a) and postmenopausal women (b).

of contralateral breast cancer as first malignant event. The secondary endpoint was the incidence of ipsilateral breast cancer reappearance, defined as local recurrence in the same quadrant or the occurrence of a second breast malignancy in different quadrants from the primary tumor. Fenretinide showed no effect on contralateral breast cancer occurrence and a nonsignificant $17 \%$ reduction in ipsilateral breast tumor reappearance. However, a different effect was noted when the analysis was stratified by menopausal status, with a beneficial trend in premenopausal women on both contralateral and ipsilateral breast cancer (38\%) and a reversed trend on contralateral breast cancer in postmenopausal women, as highlighted in Figure 3. Importantly, the protective effect persisted for up to 15 years (i.e., 10 years after retinoid cessation) [41]. Most notably, the younger the women were, the greater the benefit of fenretinide. Such benefit was associated 
with a remarkable $50 \%$ risk reduction in women aged 40 years or younger, whereas the benefit disappeared after 55 years of age. Interestingly, the incidence of ovarian cancer during the 5-year intervention period was significantly lower in the treatment arm [62]. This effect has been confirmed in an important update [63]. This phase III trial suggested a possible role of fenretinide as a preventive agent acting at different levels of breast carcinogenesis. Admittedly, the results obtained during the phase III trial on our subgroups had not been foreseen when the study was planned. While there are plausible biological explanations for this selective effect, our findings are hypothesis generating and do not have immediate practical clinical implications, although they do provide the rationale for testing the drug's efficacy in premenopausal women. Moreover, this protective effect was suggested in women with a high probability of carrying a BRCA-1 mutation. Indeed, fenretinide was highly effective in inhibiting the growth of BRCA-1 mutated breast cancer cell lines [42]. When considering the protective activity of fenretinide on second breast cancer in young women and a similar trend on ovarian cancer, at least during intervention, it appears that women with germline BRCA-1 and BRCA-2 mutations may be ideal candidates for further investigation of this drug.

\section{Future Studies}

All the collected data as well as fenretinide characteristics make this drug an excellent candidate for chemoprevention of the highlighted subgroup, that is, young healthy women with a high susceptibility to early-onset breast and ovarian cancer, such as BRCA1/2 mutation carriers or women with a significant familial risk. Since the drug activities are probably not strictly influenced by hormonal responsiveness, it may affect also hormone nonresponsive cancers. This may be very useful particularly in case of BRCA 1 mutation carriers.

Several drugs used in prevention settings are usually the same as those used for treatment (adjusting dosage and/or route of administration). This is possible because their mechanism of actions is also active on early-phase carcinogenesis and not only on the inhibition of the tumor growth. This is confirmed by the reduction of second breast cancer found in the reported studies of Veronesi et al. [40, 41]. These data make 4-HPR a surrogate marker of primary prevention and a favorable effect of the drug would provide a strong rationale for a primary prevention trial in unaffected women at high risk for breast cancer. Moreover, because its action does not seem to be influenced by the hormonal status, fenretinide might be active in hormone nonresponsive cancer prevention (as occurs in BRCA-1 mutation carriers). Although we obviously need to verify this hypothesis, we think it is an intriguing scenario that could be important in order to identify new pathways related to the efficacy of this drug.

Our Division of Cancer Prevention and Genetics at European Institute of Oncology to Milan has already activated a new phase III prevention trial addressed to this particular cohort of women.

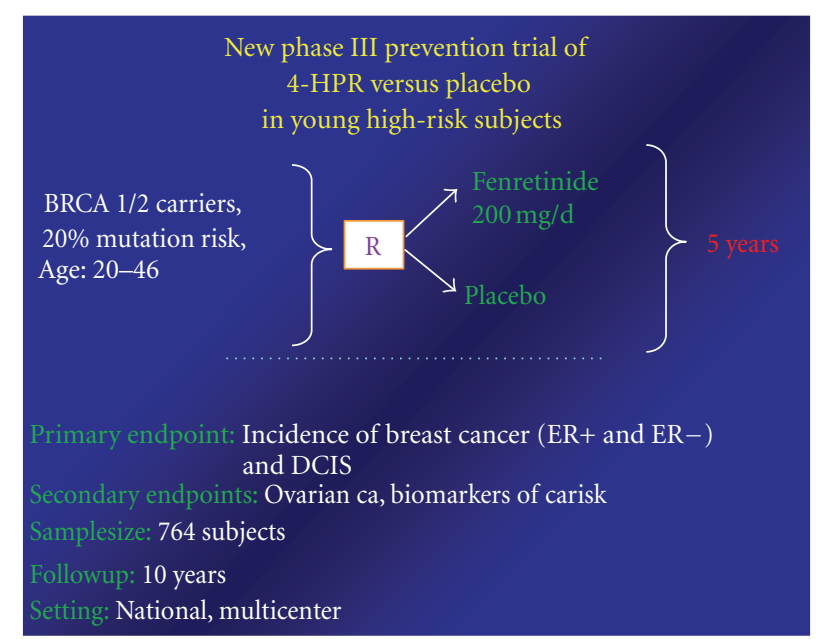

Figure 4: 4-HPR trial design.

This project is a multicentric randomized phase III placebo-controlled study. A total of 764 healthy women at increased breast cancer risk will be randomized to 4 -HPR $200 \mathrm{mg} /$ day versus placebo for 5 years. The subjects will be stratified by participating center and breast cancer risk (BRCA1 mutation versus BRCA2 versus high risk subjects). The accrual estimated time is five years. The design of the study is explained in Figure 4.

The aim of the proposed trial is to assess the efficacy of fenretinide, in reducing the incidence of breast cancer in healthy young premenopausal women at increased famil$\mathrm{ial} /$ genetic risk for breast cancer; the primary endpoint is to assess the incidence of histologically diagnosed invasive breast cancer and ductal intraepithelial neoplasia.

Secondary endpoints are the incidence of other noninvasive breast disorders (i.e., intraepithelial lobular neoplasia and atypical hyperplasia), ovarian cancer and other cancers.

Moreover, we propose an interdisciplinary research study to further investigate the mechanisms of action of fenretinide in preventing breast cancer. Early intermediate biomarkers of efficacy after 12, 36, and 60 months of treatment, genetic interactions with breast cancer risk modifiers will be explored with the primary goal to identify molecular biomarkers of response prediction. In particular, we will evaluate the percentage change in circulating biomarkers of the IGF system, androgens, retinol binding protein (RBP4 ), insulin, blood glucose, and VEGF after 12, 36, and 60 months of treatment. In a subgroup of participants, fine needle aspirate breast biopsy or cells obtained from breast ductal lavage will be drawn at baseline and after a 12-month treatment and the percentage change in RAR expression correlated with apoptosis (caspase-3) and proliferation (Ki67). Genotyping of single-nucleotide polymorphisms (SNPs) linked to breast cancer risk (MTHFR, COMT, GH, IGFBP-3, $\mathrm{AR}$, and TGF- $\beta$ genes), degree of methylation of RASSAF1 and $\operatorname{RAR} \beta$, and circulating progranulin will be assessed. The results will be correlated with mammographic instrumental measurements, plasma and tissue biomarkers after 1-year treatment. Fenretinide and its metabolites will also be 
measured to investigate drug bioavailability and compliance. Should the results of this trial confirm that fenretinide is effective in reducing breast and ovarian cancer incidence in this very high risk population, that this effect lasts for many years after treatment, and that the tolerability profile is good, we will have a further preventive chance and a new risk reduction strategy.

\section{Acknowledgments}

Special acknowledgements to Alessandra Rossi and Angela Maniscalco.

\section{References}

[1] P. Chambon, "A decade of molecular biology of retinoic acid receptors," FASEB Journal, vol. 10, no. 9, pp. 940-954, 1996.

[2] F. Rehman, P. Shanmugasundaram, and M. P. Schrey, "Fenretinide stimulates redox-sensitive ceramide production in breast cancer cells: potential role in drug-induced cytotoxicity," British Journal of Cancer, vol. 91, no. 10, pp. 1821-1828, 2004.

[3] S. Y. Sun and R. Lotan, "Retinoids and their receptors in cancer development and chemoprevention," Critical Reviews in Oncology/Hematology, vol. 41, no. 1, pp. 41-55, 2002.

[4] A. Decensi, H. Johansson, R. Miceli et al., "Long-term effects of fenretinide, a retinoic acid derivative, on the insulin-like growth factor system in women with early breast cancer," Cancer Epidemiology Biomarkers and Prevention, vol. 10, no. 10, pp. 1047-1053, 2001.

[5] Z. S. Gucev, Y. Oh, K. M. Kelley, and R. G. Rosenfeld, "Insulinlike growth factor binding protein 3 mediates retinoic acidand transforming growth factor $\beta 2$-induced growth inhibition in human breast cancer cells," Cancer Research, vol. 56, no. 7, pp. 1545-1550, 1996.

[6] I. Nunes, S. Kojima, and D. B. Rifkin, "Effects of endogenously activated transforming growth factor- $\beta$ on growth and differentiation of retinoic acid-treated HL-60 cells," Cancer Research, vol. 56, no. 3, pp. 495-499, 1996.

[7] M. Pfahl, "Nuclear receptor/AP-1 interaction," Endocrine Reviews, vol. 14, no. 5, pp. 651-658, 1993.

[8] S. Dedieu and P. Lefebvre, "Retinoids interfere with the AP1 signalling pathway in human breast cancer cells," Cellular Signalling, vol. 18, no. 6, pp. 889-898, 2006.

[9] R. C. Moon, D. L. McCormick, and R. G. Mehta, "Inhibition of carcinogenesis by retinoids," Cancer Research, vol. 43, no. 5, pp. 2469-2475, 1983.

[10] M. A. Anzano, S. W. Byers, J. M. Smith et al., "Prevention of breast cancer in the rat with 9-cis-retinoic acid as a single agent and in combination with tamoxifen," Cancer Research, vol. 54, no. 17, pp. 4614-4617, 1994.

[11] M. A. Anzano, C. W. Peer, J. M. Smith et al., "Chemoprevention of mammary carcinogenesis in the rat: combined use of raloxifene and 9-cis-retinoic acid," Journal of the National Cancer Institute, vol. 88, no. 2, pp. 123-125, 1996.

[12] K. Wu, H. T. Kim, J. L. Rodriquez et al., "Suppression of mammary tumorigenesis in transgenic mice by the RXRselective retinoid, LGD1069," Cancer Epidemiology Biomarkers and Prevention, vol. 11, no. 5, pp. 467-474, 2002.

[13] K. Wu, H. T. Kim, J. L. Rodriquez et al., "9-cis-Retinoic acid suppresses mammary tumorigenesis in C3(1)-simian virus 40 T antigen-transgenic mice," Clinical Cancer Research, vol. 6, no. 9, pp. 3696-3704, 2000.
[14] K. Wu, Y. Zhang, X. C. Xu et al., "The retinoid X receptorselective retinoid, LGD1069, prevents the development of estrogen receptor-negative mammary tumors in transgenic mice," Cancer Research, vol. 62, no. 22, pp. 6376-6380, 2002.

[15] M. M. Gottardis, E. D. Bischoff, M. A. Shirley, M. A. Wagoner, W. W. Lamph, and R. A. Heyman, "Chemoprevention of mammary carcinoma by LGD1069 (Targretin): an RXRselective ligand," Cancer Research, vol. 56, no. 24, pp. 55665570, 1996.

[16] S. Y. Sun, W. Li, P. Yue, S. M. Lippman, W. K. Hong, and R. Lotan, "Mediation of N-(4-hydoxyphenyl)retinamideinduced apoptosis in human cancer cells by different mechanisms," Cancer Research, vol. 59, no. 10, pp. 2493-2498, 1999.

[17] M. S. Sheikh, Z. M. Shao, X. S. Li et al., "N-(4hydroxyphenyl)retinamide (4-HPR)-mediated biological actions involve retinoid receptor-independent pathways in human breast carcinoma," Carcinogenesis, vol. 16, no. 10, pp. 2477-2486, 1995.

[18] A. N. Fanjul, D. Delia, M. A. Pierotti, D. Rideout, J. Q. Qiu, and M. Pfahl, "4-Hydroxyphenyl retinamide is a highly selective activator of retinoid receptors," Journal of Biological Chemistry, vol. 271, no. 37, pp. 22441-22446, 1996.

[19] V. Giandomenico, F. Andreola, M. L. Rodriguez de la Concepcion, S. J. Collins, and L. M. de Luca, "Retinoic acid and 4hydroxyphenylretinamide induce growth inhibition and tissue transglutaminase through different signal transduction pathways in mouse fibroblasts (NIH 3T3 cells)," Carcinogenesis, vol. 20, no. 6, pp. 1133-1135, 1999.

[20] A. L. Anding, J. S. Chapman, D. W. Barnett, R. W. Curley Jr., and M. Clagett-Dame, "The unhydrolyzable fenretinide analogue 4-hydroxybenzylretinone induces the proapoptotic genes GADD153 (CHOP) and Bcl-2-binding component 3 (PUMA) and apoptosis that is caspase-dependent and independent of the retinoic acid receptor," Cancer Research, vol. 67, no. 13, pp. 6270-6277, 2007.

[21] D. D’Elia, A. Aiello, L. Lombardi et al., “N-(4Hydroxyphenyl)retinamide induces apoptosis of malignant hemopoietic cell lines including those unresponsive to retinoic acid," Cancer Research, vol. 53, no. 24, pp. 6036-6041, 1993.

[22] R. Lotan, "Retinoids and apoptosis: implications for cancer chemoprevention and therapy," Journal of the National Cancer Institute, vol. 87, no. 22, pp. 1655-1657, 1995.

[23] W. F. Holmes, D. R. Soprano, and K. J. Soprano, "Comparison of the mechanism of induction of apoptosis in ovarian carcinoma cells by the conformationally restricted synthetic retinoids CD437 and 4-HPR," Journal of Cellular Biochemistry, vol. 89, no. 2, pp. 262-278, 2003.

[24] W. F. Holmes, D. R. Soprano, and K. J. Soprano, "Synthetic retinoids as inducers of apoptosis in ovarian carcinoma cell lines," Journal of Cellular Physiology, vol. 199, no. 3, pp. 317329, 2004.

[25] V. Appierto, P. Tiberio, E. Cavadini, P. Casalini, G. Cappelletti, and F. Formelli, "Antimitotic effect of the retinoid 4-oxofenretinide through inhibition of tubulin polymerization: a novel mechanism of retinoid growth-inhibitory activity," Molecular Cancer Therapeutics, vol. 8, no. 12, pp. 3360-3368, 2009.

[26] N. Oridate, S. Suzuki, M. Higuchi, M. F. Mitchell, W. K. Hong, and R. Lotan, "Involvement of reactive oxygen species in $\mathrm{N}$ (4- hydroxyphenyl)retinamide-induced apoptosis in cervical carcinoma cells," Journal of the National Cancer Institute, vol. 89, no. 16, pp. 1191-1198, 1997.

[27] D. Delia, A. Aiello, L. Meroni, M. Nicolini, J. C. Reed, and M. A. Pierotti, "Role of antioxidants and intracellular free radicals 
in retinamide-induced cell death," Carcinogenesis, vol. 18, no. 5, pp. 943-948, 1997.

[28] F. Tosetti, R. Venè, G. Arena et al., "N-(4-hydroxyphenyl) retinamide inhibits retinoblastoma growth through reactive oxygen species-mediated cell death," Molecular Pharmacology, vol. 63, no. 3, pp. 565-573, 2003.

[29] B. J. Maurer, L. Melton, C. Billups, M. C. Cabot, and C. P. Reynolds, "Synergistic cytotoxicity in solid tumor cell lines between $\mathrm{N}$-(4-hydroxyphenyl)retinamide and modulators of ceramide metabolism," Journal of the National Cancer Institute, vol. 92, no. 23, pp. 1897-1909, 2000.

[30] M. B. Sporn, N. M. Dunlop, D. L. Newton, and J. M. Smith, "Prevention of chemical carcinogenesis by vitamin A and its synthetic analogs (retinoids)," Federation Proceedings, vol. 35, no. 6, pp. 1332-1338, 1976.

[31] R. C. Moon, H. J. Thompson, P. J. Becci et al., "N-(4hydroxyphenyl)retinamide, a new retinoid for prevention of breast cancer in the rat," Cancer Research, vol. 39, no. 4, pp. 1339-1346, 1979.

[32] G. J. Kelloff, J. A. Crowell, C. W. Boone et al., "Clinical development plan: N-(4-hydroxyphenyl)retinamide," Journal of cellular biochemistry. Supplement, vol. 20, pp. 176-196, 1994.

[33] R. G. Mehta, R. C. Moon, M. Hawthorne, F. Formelli, and A. Costa, "Distribution of fenretinide in the mammary gland of breast cancer patients," European Journal of Cancer, vol. 27, no. 2, pp. 138-141, 1991.

[34] J. S. Chapman, K. L. Weiss, R. W. Curley Jr., M. A. Highland, and M. Clagett-Dame, "Hydrolysis of 4-HPR to atRA occurs in vivo but is not required for retinamide-induced apoptosis," Archives of Biochemistry and Biophysics, vol. 419, no. 2, pp. 234-243, 2003.

[35] C. P. Reynolds, K. K. Matthay, J. G. Villablanca, and B. J. Maurer, "Retinoid therapy of high-risk neuroblastoma," Cancer Letters, vol. 197, no. 1-2, pp. 185-192, 2003.

[36] P. H. O’Donnell, W. X. Guo, C. P. Reynolds, and B. J. Maurer, " $\mathrm{N}$-(4-hydroxyphenyl)retinamide increases ceramide and is cytotoxic to acute lymphoblastic leukemia cell lines, but not to non-malignant lymphocytes," Leukemia, vol. 16, no. 5, pp. 902-910, 2002.

[37] S. Batra, C. P. Reynolds, and B. J. Maurer, "Fenretinide cytotoxicity for Ewing's sarcoma and primitive neuroectodermal tumor cell lines is decreased by hypoxia and synergistically enhanced by ceramide modulators," Cancer Research, vol. 64, no. 15, pp. 5415-5424, 2004.

[38] A. Costa, W. Malone, M. Perloff et al., "Tolerability of the synthetic retinoid fenretinide ${ }^{\circledR}$ (HPR)," European Journal of Cancer and Clinical Oncology, vol. 25, no. 5, pp. 805-808, 1989.

[39] F. Formelli, R. Carsana, A. Costa et al., "Plasma retinol level reduction by the synthetic retinoid fenretinide: a one year follow-up study of breast cancer patients," Cancer Research, vol. 49, no. 21, pp. 6149-6152, 1989.

[40] U. Veronesi, G. de Palo, E. Marubini et al., "Randomized trial of fenretinide to prevent second breast malignancy in women with early breast cancer," Journal of the National Cancer Institute, vol. 91, no. 21, pp. 1847-1856, 1999.

[41] U. Veronesi, L. Mariani, A. Decensi et al., "Fifteen-year results of a randomized phase III trial of fenretinide to prevent second breast cancer," Annals of Oncology, vol. 17, no. 7, pp. 10651071, 2006.

[42] A. M. Simeone, C. X. Deng, G. J. Kelloff, V. E. Steele, M. M. Johnson, and A. M. Tari, "N-(4-hydroxyphenyl)retinamide is more potent than other phenylretinamides in inhibiting the growth of BRCA1-mutated breast cancer cells," Carcinogenesis, vol. 26, no. 5, pp. 1000-1007, 2005.
[43] R. Baserga, "The insulin-like growth factor I receptor: a key to tumor growth?” Cancer Research, vol. 55, no. 2, pp. 249-252, 1995.

[44] S. E. Hankinson, W. C. Willett, G. A. Colditz et al., "Circulating concentrations of insulin-like growth factor-I and risk of breast cancer," Lancet, vol. 351, no. 9113, pp. 1393-1396, 1998.

[45] J. M. Chan, M. J. Stampfer, E. Giovannucci et al., "Plasma insulin-like growth factor-I and prostate cancer risk: a prospective study," Science, vol. 279, no. 5350, pp. 563-566, 1998.

[46] H. Yu, M. R. Spitz, J. Mistry, J. Gu, W. K. Hong, and X. Wu, "Plasma levels of insulin-like growth factor-I and lung cancer risk: a case-control analysis," Journal of the National Cancer Institute, vol. 91, no. 2, pp. 151-156, 1999.

[47] J. Ma, M. N. Pollak, E. Giovannucci et al., "Prospective study of colorectal cancer risk in men and plasma levels of insulin-like growth factor (IGF)-I and IGF-binding protein-3," Journal of the National Cancer Institute, vol. 91, no. 7, pp. 620-625, 1999.

[48] R. E. Favoni, A. de Cupis, S. Bruno et al., "Modulation of the insulin-like growth factor-I system by $\mathrm{N}$-(4-hydroxyphenyl)retinamide in human breast cancer cell lines," British Journal of Cancer, vol. 77, no. 12, pp. 2138-2147, 1998.

[49] R. Torrisi, F. Pensa, M. A. Orengo et al., "The synthetic retinoid fenretinide lowers plasma insulin-like growth factor-I levels in breast cancer patients," Cancer Research, vol. 53, no. 20, pp. 4769-4771, 1993.

[50] A. M. Simeone, Y. J. Li, L. D. Broemeling, M. M. Johnson, M. Tuna, and A. M. Tari, "Cyclooxygenase-2 Is Essential for HER2/neu to Suppress N-(4-Hydroxyphenyl)retinamide apoptotic effects in breast cancer cells," Cancer Research, vol. 64, no. 4, pp. 1224-1228, 2004.

[51] T. Camerini, L. Mariani, G. de Palo et al., "Safety of the synthetic retinoid fenretinide: long-term results from a controlled clinical trial for the prevention of contralateral breast cancer," Journal of Clinical Oncology, vol. 19, no. 6, pp. 1664-1670, 2001.

[52] A. Decensi, R. Torrisi, A. Gozza et al., "Effect of fenretinide on bone mineral density and metabolism in women with early breast cancer," Breast Cancer Research and Treatment, vol. 53, no. 2, pp. 145-151, 1999.

[53] J. Zujewski, L. Pai, L. Wakefield et al., "Tamoxifen and fenretinide in women with metastatic breast cancer," Breast Cancer Research and Treatment, vol. 57, no. 3, pp. 277-283, 1999.

[54] M. A. Cobleigh, K. Dowlatshahi, T. A. Deutsch et al., "Phase I/II trial of tamoxifen with or without fenretinide, an analog of vitamin A, in women with metastatic breast cancer," Journal of Clinical Oncology, vol. 11, no. 3, pp. 474-477, 1993.

[55] D. R. Doose, F. L. Minn, S. Stellar, and R. K. Nayak, "Effects of meals and meal composition on the bioavailability of fenretinide," Journal of Clinical Pharmacology, vol. 32, no. 12, pp. 1089-1095, 1992.

[56] F. Formelli, M. Clerici, T. Campa et al., "Five-year administration of fenretinide: pharmacokinetics and effects on plasma retinol concentrations," Journal of Clinical Oncology, vol. 11, no. 10, pp. 2036-2042, 1993.

[57] Y. M. Peng, W. S. Dalton, D. S. Alberts, M. J. Xu, H. Lim, and F. L. Meyskens, "Pharmacokinetics of N-4-hydroxyphenylretinamide and the effect of its oral administration on plasma retinol concentrations in cancer patients," International Journal of Cancer, vol. 43, no. 1, pp. 22-26, 1989.

[58] T. A. Ratko, C. J. Detrisac, N. M. Dinger, C. F. Thomas, G. J. Kelloff, and R. C. Moon, "Chemopreventive efficacy of combined retinoid and tamoxifen treatment following 
surgical excision of a primary mammary cancer in female rats," Cancer Research, vol. 49, no. 16, pp. 4472-4476, 1989.

[59] B. Conley, J. O'Shaughnessy, S. Prindiville et al., "Pilot trial of the safety, tolerability, and retinoid levels of N-(4hydroxyphenyl) retinamide in combination with tamoxifen in patients at high risk for developing invasive breast cancer," Journal of Clinical Oncology, vol. 18, no. 2, pp. 275-283, 2000.

[60] A. Decensi, C. Robertson, A. Guerrieri-Gonzaga et al., "Randomized double-blind 2 X 2 trial of low-dose tamoxifen and fenretinide for breast cancer prevention in high-risk premenopausal women," Journal of Clinical Oncology, vol. 27, no. 23, pp. 3749-3756, 2009.

[61] A. Decensi, B. Bonanni, L. Baglietto et al., "A two-by-two factorial trial comparing oral with transdermal estrogen therapy and fenretinide with placebo on breast cancer biomarkers," Clinical Cancer Research, vol. 10, no. 13, pp. 4389-4397, 2004.

[62] G. de Palo, U. Veronesi, T. Camerini et al., "Can fenretinide protect women against ovarian cancer?" Journal of the National Cancer Institute, vol. 87, no. 2, pp. 146-147, 1995.

[63] G. de Palo, L. Mariani, T. Camerini et al., "Effect of fenretinide on ovarian carcinoma occurrence," Gynecologic Oncology, vol. 86, no. 1, pp. 24-27, 2002. 



Submit your manuscripts at

http://www.hindawi.com
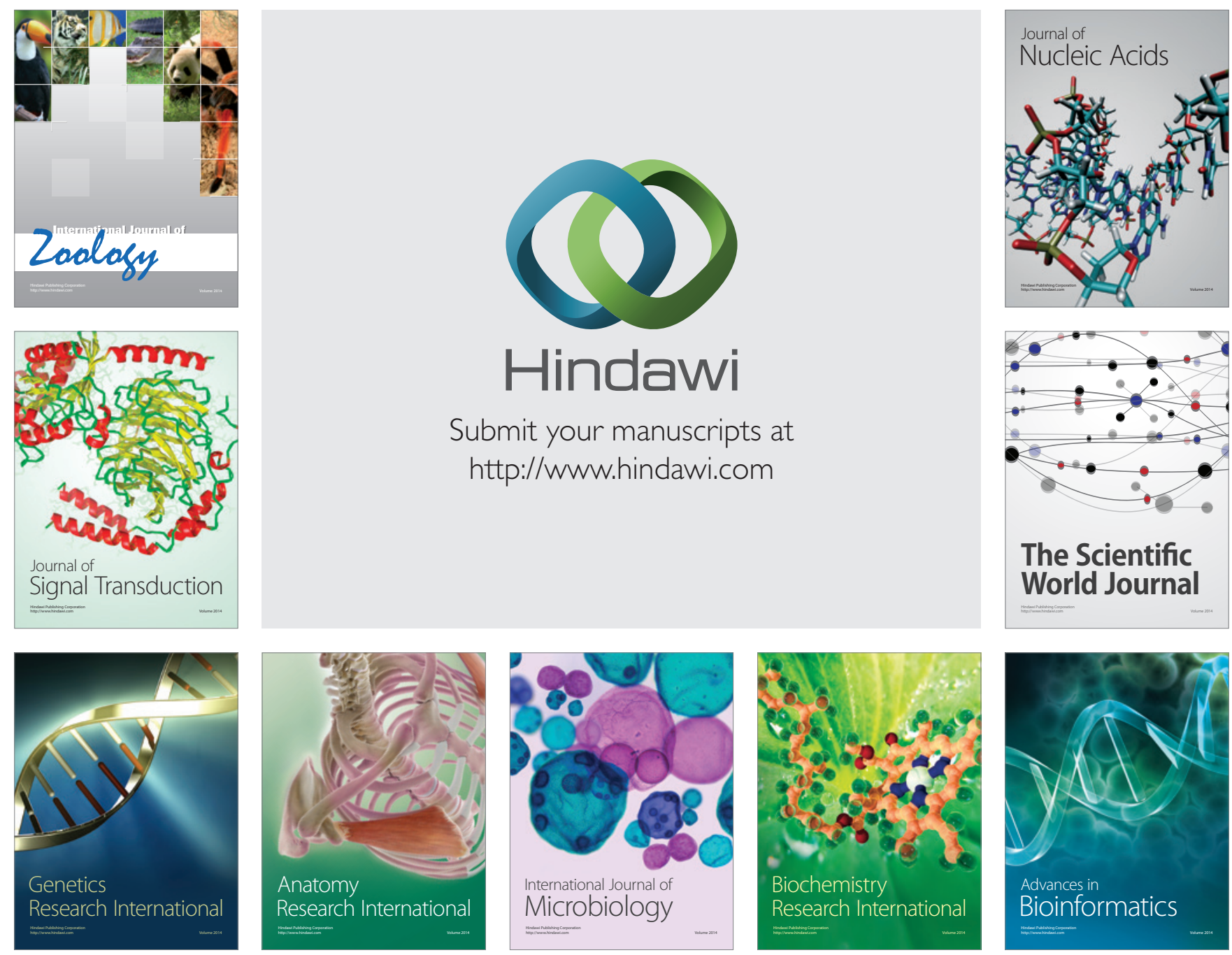

The Scientific World Journal
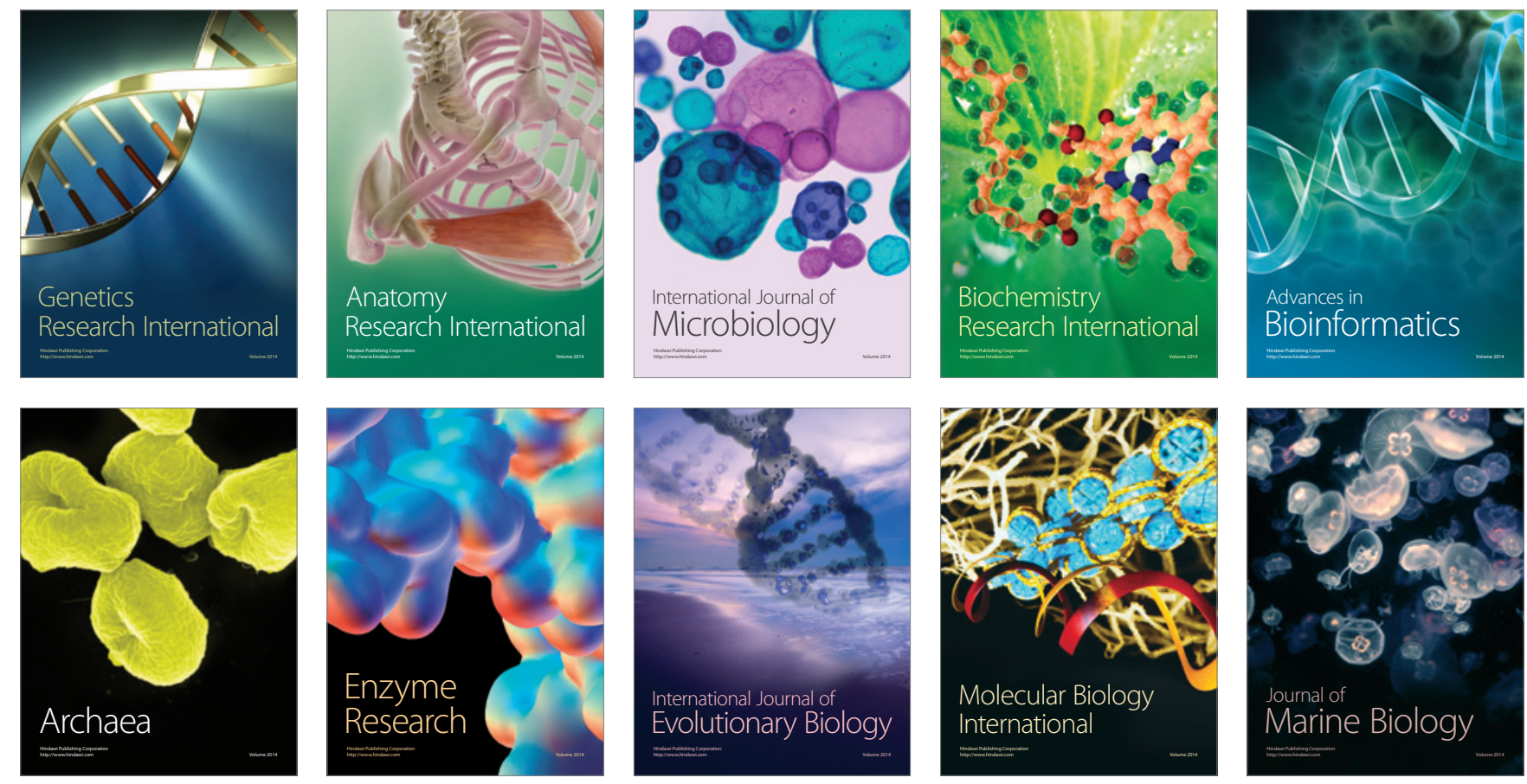